\title{
Central skull base osteomyelitis due to nasopharyngeal Klebsiella infection
}

\author{
Kyoung-Nam Woo, MD'; Jieun Roh, MD²; Seung-Kug Baik, $\mathrm{PhD}^{2}$; \\ Dong-Hyeon Shin, MD'; Ki-Seok Park, MD'; Min-Gyu Park, PhD';

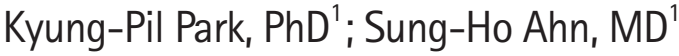

'Department of Neurology, Research Institute for Convergence of Biomedical Science and Technology, Pusan National University Yangsan Hospital, Pusan National University School of Medicine, Busan, Republic of Korea

${ }^{2}$ Department of Radiology, Research Institute for Convergence of Biomedical Science and Technology, Pusan National University Yangsan Hospital, Pusan National University School of Medicine, Busan, Republic of Korea

\section{Journal of Neurocritical \\ CASE REPORT \\ Received: July 25, 2020 \\ Revised: September 4, 2020 \\ Accepted: September 6, 2020 \\ Corresponding Author: \\ Sung-Ho Ahn, MD \\ Department of Neurology, Pusan \\ National University Yangsan Hospital, \\ Pusan National University School of \\ Medicine, 20 Geumo-ro, Mulgeum- \\ eup, Yangsan 50612, Korea \\ Tel: +82-55-360-2122 \\ Fax: +82-55-360-2152 \\ E-mail: caesara@pnuyh.co.kr}

Background: Skull base osteomyelitis (SBO) is a rare but life-threatening disease occurring as a complication of malignant otitis externa, and accompanied by venous sinus thrombosis, meningitis, abscess, cranial neuropathies, and carotid invasion as complications. Central SBO may originate from a paranasal infection, such as sphenoidal or ethmoidal sinusitis without associated external otitis.

Case Report: We describe a 36-year-old Sri Lankan male with central SBO presenting with multiple embolic infarctions and meningitis caused by a nasopharyngeal Klebsiella infection that had invaded the left internal carotid artery. Despite complications, such as endogenous endophthalmitis, abscesses in the brain parenchyma, and mycotic aneurysms in cerebral vessels, the patient recovered after 8 weeks of intensive antibiotics treatment except for a remnant mycotic aneurysmal dilatation in the internal carotid artery.

Conclusion: This is the first report of central SBO caused by a nasopharyngeal Klebsiella infection, which invaded the left internal carotid artery and led to multiple complications.

Keywords: Skull base osteomyelitis; Infarction; Klebsiella

\section{INTRODUCTION}

Skull base osteomyelitis (SBO) is a rare but life-threatening disease usually seen as a complication of otitis externa [1]. It initially involves the lateral part of the temporal bone and extends to the petrous apex and clivus [2], accompanied by bone erosion, thrombophlebitis, and hematogenous seeding [3]. Thus, patients with SBO typically present with otalgia, otorrhea, hearing loss, and cranial nerve dysfunction in the immunocompromised, particularly older diabetic patients [4].
Although SBO typically evolves as a complication of malignant otitis externa, atypical or central SBO without associated external otitis has occasionally been reported $[5,6]$. Central SBO is generally caused by paranasal infections, such as sphenoidal or ethmoidal sinusitis; thus, patients with central SBO usually experience headaches, atypical facial pain, and cranial nerve palsies at the time of initial presentation $[7,8]$. Furthermore, compared to the known complications of $\mathrm{SBO}$, including venous sinus thrombosis, meningitis, abscess, cranial neuropathies, and carotid invasion with or without ischemic stroke [1], the pattern of complica-

C 2020 The Korean Neurocritical Care Society

This is an Open Access article distributed under the terms of the Creative Commons Attribution Non-Commercial License (http://creativecommons.org/licenses/by-nc/4.0/) which permits unrestricted noncommercial use, distribution, and reproduction in any medium, provided the original work is properly cited. 
tions in central SBO is different. This is because the inflammation in the cavernous sinus can easily spread to the carotid siphon, causing inflammatory arteritis and, in turn, leading to stroke by carotid artery occlusion or septic embolism [9].

We describe a case of central SBO who initially presented with severe headache, fever, aphasia, and right hemiparesis due to multiple embolic infarctions and meningitis, caused by a nasopharyngeal Klebsiella infection that invaded the left internal carotid artery (ICA), which led to septic embolism and mycotic aneurysmal formation.

\section{CASE REPORT}

A 36-year-old Sri Lankan male was transferred to our emergency department with the chief complaints of headache, fever, and aphasia emerging after 3 weeks of sore throat and cough without a preceding history of otitis externa. He had been diagnosed with type 2 diabetes mellitus 3 years before but had discontinued medication 3 months prior to the current presentation. On neurological examination, his mental status was alert, and cranial nerve examinations revealed unremarkable findings. However, he showed global aphasia and right hemiparesis, equivalent to a score of 9 points in the National Institutes of Health Stroke Scale (NIHSS), with neck stiffness. Otorhinolaryngological examination revealed left nasopharyngeal mucosal swelling with purulent discharge and otitis media with effusion. Laboratory data showed elevated leukocyte count $(15,150 / \mu \mathrm{L})$, erythrocyte sedimentation rate (ESR; $105 \mathrm{~mm} / \mathrm{hr}$ ), C-reactive protein (CRP; $15.76 \mathrm{mg} / \mathrm{dL}$ ), and glycated hemoglobin (12.3\%).

Brain magnetic resonance imaging (MRI) showed multiple embolic infarctions, particularly in the territory of the left middle cerebral artery, and abnormally increased gadolinium enhancement in the left parapharyngeal space invading the left infratemporal fossa and skull base. In addition, increased sulcal effacement suspicious of leptomeningitis was detected in both frontal and parietal lobes. Computed tomographic angiography revealed luminal narrowing with filling defects in the petrous portion of the left ICA (Fig. 1A and B). These findings were compatible with the invasion of nasopharyngeal infection into the carotid artery and skull base, which, in turn, led to multiple embolic cerebral infarctions. Cultures of peripheral blood and nasopharyngeal discharges revealed the presence of Klebsiella pneumoniae. Thus, the patient was initially treated with intravenous antibiotics, including ceftazidime and vancomycin as the empirical regimen and then treated with a modified regimen, including ceftriaxone and vancomycin targeting the Klebsiella for 8 weeks of schedule.

On the 3rd day after admission, the patient suddenly developed a blurring vision accompanied by eyeball pain, chemosis, and conjunctival injection in his left eye. On ophthalmologic examination, bacterial endophthalmitis was diagnosed due to septic embolism from the invaded ICA. Subsequently, intravitreal injection of vancomycin and ceftazidime was administered and vitrectomy was performed. Follow-up brain MRI and magnetic resonance angiography (MRA) after 2 weeks of treatment showed a newly developed brain abscess within prior ischemic lesions and mycotic aneurysmal formation in the left cervical ICA (Fig. 1C). Sequential follow-up of brain MRI and MRA at 4 weeks and 8 weeks of antibiotic treatment showed an improving course of prior lesions with a decreased size of brain abscess and decreased gadolinium enhancement in the left infratemporal fossa and skull base, but an increased size of the aneurysm in the left cervical ICA (Fig. 1D). After completing 8 weeks of intravenous antibiotic treatment, the patient showed an improvement in neurological deficit except for a remnant mild degree of facial palsy (NIHSS 1) with normalized serum levels of ESR and CRP and non-detectable pathogen on repeatedly performed blood cultures. Although we considered a rescue treatment with stent-assisted coil embolization to manage the worsening aneurysmal dilation in the left ICA, the patient was required to return to his own country due to the expiration of his visa.

\section{DISCUSSION}

We describe a case of a 36-year-old Sri Lankan male with central SBO caused by a nasopharyngeal Klebsiella infection that invaded the left ICA and led to multiple embolic infarctions and meningitis, followed by endogenous endophthalmitis, brain abscess, and mycotic aneurysms despite antibiotic treatment. Central SBO originating from nasopharyngeal infection is rarely reported and difficult to diagnose as the radiological findings frequently mimic nasopharyngeal carcinoma with skull base involvement [7]. SBO is a rare but life-threatening disease if not properly diagnosed and treated. A recent systematic retrospective study showed that 42 patients with central SBO had a $9.5 \%$ mortality rate, with longterm neurologic sequelae seen in an additional $31 \%$ of cases despite aggressive treatment $[5,10]$. Therefore, prompt commencement and selection of appropriate antibiotics are crucial for the treatment of central SBO, and close monitoring is essential for early detection of multiple complications, such as septic embolism and mycotic aneurysmal formation even during the antibiotic treatment.

Central SBO mainly involves the sphenoid and occipital bones and usually occurs due to the direct spread of infection from the paranasal sinuses, mastoid process, middle ear, or oral cavity [10]. 

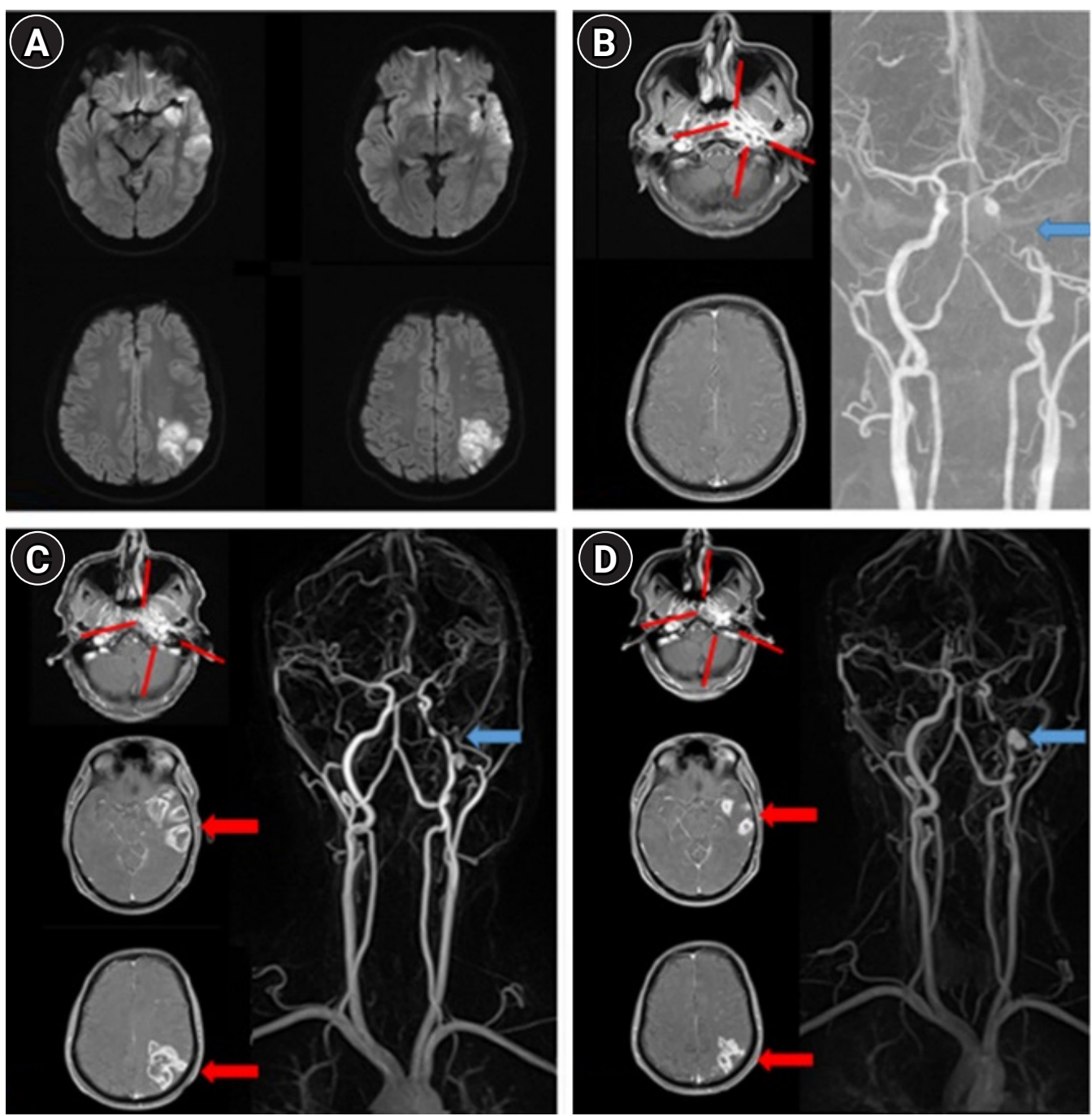

Fig. 1. Brain magnetic resonance imaging (MRI) and computed tomography angiography (CTA) findings at admission (A, B), at 2 weeks of hospitalization after the onset of endogenous endophthalmitis (C), and at 8 weeks of hospitalization after completion of the antibiotic treatment (D). (A) Baseline MRI revealed multiple embolic infarctions on diffusion weighted image sequence in the region of the left middle cerebral artery. (B) Baseline MRI revealed an increased gadolinium enhancement in the left infratemporal fossa and skull base (red lines) and sulcal enhancement in the hemispheres (left), and baseline CTA (right) revealed a narrowing in the left petrous portion of the internal carotid artery (ICA; blue arrow), compatible with osteomyelitis due to the extension of a deep neck infection into the ICA. (C) Follow-up MRI revealed an increased enhancement in the left infratemporal fossa and skull base (red lines) and newly developed ring enhancement in the infarct area (suspicious of brain abscess formation; red arrows), and follow-up magnetic resonance angiography (MRA) revealed an interval improvement of the left distal ICA stenosis with a newly developed aneurysmal formation (mycotic aneurysm, blue arrow). (D) Follow-up MRI revealed an improvement in the inflammation in the left skull base and neck (red lines) and brain abscess (red arrow), and follow-up MRA revealed an increased size of the aneurysm in the ICA (blue arrow).

Underlying diabetes mellitus and immunodeficiency are common predisposing factors [4]. Headache and/or facial pain and cranial nerve palsies are common at initial presentation, followed by nasal congestion/discharge and fever. According to the previous literature, Pseudomonas aeruginosa is the most common pathogen causing central SBO, while fungal or mixed bacterial infections have also been reported [7]. Although standard protocol has not been proposed regarding the duration of antibiotic therapy in central SBO, currently, 6-20 weeks of broad-spectrum antimicrobial agents are recommended [11]. Our patient also recovered after 8 weeks of intensive antibiotic treatment with ceftriaxone and vancomycin targeting the Klebsiella infection. Central SBO caused by Klebsiella has been reported in patients with sphenoid sinus cholesteatoma [12] and a history of previous ear infections [11,13]. Thus, to our knowledge, this is the first report of a central SBO case caused by nasopharyngeal Klebsiella infection. In this case, the nasopharyngeal Klebsiella infection led to SBO by invading the ICA, which, in turn, led to multiple embolic infarctions, men- 
ingitis, endophthalmitis due to septic embolism, and mycotic aneurysms.

\section{ARTICLE INFORMATION}

\section{Ethics statement}

Approval for this study was waived by the Institutional Review Board of Pusan National University Yangsan Hospital because of the usual practice, and informed consent was obtained from the patient to publish his case.

\section{Conflict of interest}

Dr. SH Ahn is an editorial board member of the journal but did not involve in the peer reviewer selection, evaluation, or decision process of this article. No other potential conflicts of interest relevant to this article were reported.

\section{Funding}

This study was supported by a 2020 research grant from Pusan National University Yangsan Hospital.

\section{ORCID}

Kyoung-Nam Woo

Jieun Roh

Seung-Kug Baik

Dong-Hyeon Shin

Ki-Seok Park

Min-Gyu Park

Kyung-Pil Park

Sung-Ho Ahn

https://orcid.org/0000-0001-8115-0905 https://orcid.org/0000-0002-6876-3990 https://orcid.org/0000-0001-5408-747X https://orcid.org/0000-0002-0108-3675 https://orcid.org/0000-0002-4034-2780 https://orcid.org/0000-0003-2968-6328 https://orcid.org/0000-0003-4952-3796 https://orcid.org/0000-0002-8376-545X

\section{Author contributions}

Conceptualization: KNW, SHA. Formal analysis: all authors. Funding acquisition: SHA. Project administration: SHA. Writing-original draft, review \& editing: all authors.

\section{REFERENCES}

1. Severino M, Liyanage S, Novelli V, Cheesborough B, Saunders D, Gunny R, et al. Skull base osteomyelitis and potential cerebrovascular complications in children. Pediatr Radiol 2012;
42:867-74.

2. Azizi SA, Fayad PB, Fulbright R, Giroux ML, Waxman SG. Clivus and cervical spinal osteomyelitis with epidural abscess presenting with multiple cranial neuropathies. Clin Neurol Neurosurg 1995;97:239-44.

3. Rubin Grandis J, Branstetter BF 4th, Yu VL. The changing face of malignant (necrotising) external otitis: clinical, radiological, and anatomic correlations. Lancet Infect Dis 2004;4:34-9.

4. Rothholtz VS, Lee AD, Shamloo B, Bazargan M, Pan D, Djalilian HR. Skull base osteomyelitis: the effect of comorbid disease on hospitalization. Laryngoscope 2008; 118:1917-24.

5. Johnson AK, Batra PS. Central skull base osteomyelitis: an emerging clinical entity. Laryngoscope 2014;124:1083-7.

6. Clark MP, Pretorius PM, Byren I, Milford CA. Central or atypical skull base osteomyelitis: diagnosis and treatment. Skull Base 2009; 19:247-54.

7. Kilich E, Dwivedi R, Segal S, Jayawant S, Sadarangani M. Symptomatic stroke complicating central skull base osteomyelitis following otitis media in a 2-year old boy: case report and review of the literature. Int J Pediatr Otorhinolaryngol 2016;89:140-4.

8. Mani N, Sudhoff H, Rajagopal S, Moffat D, Axon PR. Cranial nerve involvement in malignant external otitis: implications for clinical outcome. Laryngoscope 2007;117:907-10.

9. Hashimoto Y, Tateishi T. Atypical skull base osteomyelitis suspected of spreading inflammation from the ear canal with unilateral multiple cranial neuropathy and cerebral infarctions. Rinsho Shinkeigaku 2019;59:205-10.

10. Ridder GJ, Breunig C, Kaminsky J, Pfeiffer J. Central skull base osteomyelitis: new insights and implications for diagnosis and treatment. Eur Arch Otorhinolaryngol 2015;272:1269-76.

11. Muranjan SN, Khadilkar SV, Wagle SC, Jaggi ST. Central skull base osteomyelitis: diagnostic dilemmas and management issues. Indian J Otolaryngol Head Neck Surg 2016;68:149-56.

12. Kanjanawasee D, Chaowanapanja P, Keelawat S, Snidvongs K. Sphenoid sinus cholesteatoma-complications and skull base osteomyelitis: case report and review of literature. Clin Med Insights Case Rep 2019;12:1179547619835182.

13. Low WK, Lhu HL. Skull base osteomyelitis from otitis media presenting as the collet-sicard syndrome. Case Rep Otolaryngol 2018;2018:1407417. 